\title{
Capsule Commentary on Turner et al., Randomized Trial of Chronic Pain Self-management Program in Community or Clinic for Low-Income Primary Care Patients
}

\author{
Lauren S. Penney, Ph.D. ${ }^{1,2}$ \\ ${ }^{1}$ South Texas Veterans Health Care System, San Antonio, TX, USA; ²University of Texas Health San Antonio, San Antonio, TX, USA.
}

J Gen Intern Med 33(5):746

DOI: $10.1007 / \mathrm{s} 11606-018-4370-5$

(c) Society of General Internal Medicine (outside the USA) 2018

$\mathrm{T}$ his parallel group trial by Turner et al. ${ }^{1}$ evaluated two tailored interventions for low-income, mostly Hispanic chronic pain patients who had been prescribed opioids for more than 2 months. Participants were randomized to either to (1) nine group community lectures or (2) monthly one-onone meetings with community health workers. At the end of the intervention (6 months), subjects in both community and clinic experienced improvement in multiple functional outcome measures, though only those receiving one-on-one meetings saw significant improvement in the 6-min walk test and SF-12 physical component scale in ITT analyses.

Participants in this study are from communities with limited access to pain management resources. Patient, provider, health care system, and other social structural factors contribute to inequities in pain treatment and outcomes. ${ }^{2}$ It is clear that reducing disparities will require multifactorial approaches. ${ }^{3,4}$ Patient-level interventions cannot use education alone but must include other qualities which are both practical to implement and sensitive to local values and needs. ${ }^{5}$ Functional restoration training using trained community health workers and other types of coaches potentially offers a low-cost, simple intervention in communities like these and increases access to pain treatment.

The interventions draw strength from tailoring based on principles from community-based participatory research, using bilingual staff and one-on-one coaching, and allowing flexibility in implementation to encourage participant completion. These qualities help build relationships and trust and focus on meeting community needs. Unfortunately, we do not have data regarding patient perceptions or satisfaction with

Published online February 27, 2018 the interventions, or patient outcomes beyond the end of the intervention period. Sustainment of outcomes poses a formidable challenge for short-term interventions addressing chronic pain. Sufferers must learn how to self-manage conditions in which pain levels and functional conditioning, and the biopsychosocial factors which affect them, shift over time.

Increasingly, clinicians and health systems are approaching chronic pain from biopsychosocial perspectives. In this model, functional restoration is an important component to help support people who live with chronic pain. Studies like this offer new options for implementing solutions to facilitate pain selfmanagement in lower-resourced communities.

Corresponding Author: Lauren S. Penney, Ph.D.; South Texas Veterans Health Care System, San Antonio, TX, USA (e-mail: Lauren. Penney@va.gov).

Compliance with ethical standards:

Conflict of interest: The author declares that she has no conflicts of interest.

\section{REFERENCES}

1. Turner BJ, Liang Y, Simmonds MJ, Rodriguez N, Bobadilla Y, Yin Z. Randomized trial of chronic pain self-management program in community or clinic for low-income primary care patients. J Gen Intern Med https:// doi.org/10.1007/s11606-017-4244-2

2. Green CR, Anderson KO, Baker TA, et al. The Unequal Burden of Pain: Confronting Racial and Ethnic Disparities in Pain. Pain Med 2003;4(3):277-294.

3. Washington DL, Bowles J, Saha S, et al. Transforming Clinical Practice to Eliminate Racial-Ethnic Disparities in Healthcare. J Gen Intern Med 2008;23(5):685-691.

4. Meghani SH, Polomano RC, Tait RC, Vallerand AH, Anderson Ko, Gallagher RM. Advancing a National Agenda to Eliminate Disparities in Pain Care: Directions for Health Policy, Education, Practice, and Research. Pain Med 2012;13(1):5-28.

5. Anderson KO, Green CR, Payne R. Racial and Ethnic Disparities in Pain: Causes and Consequences of Unequal Care. J Pain 2009;10(12):11871204. 